\title{
Ordering transformation in icosahedral quasicrystals and related crystalline phases
}

\author{
N K MUKHOPADHYAY ${ }^{\dagger}$, K CHATTOPADHYAY* and \\ S RANGANATHAN \\ Department of Metallurgy, Indian Institute of Science, Bangalore 560012, India \\ 'National Metallurgical Laboratory. Jamshedpur 831 007. India \\ MS received 18 October 1994; revised 29 June 1996
}

\begin{abstract}
Arcs of diffuse intensity appear in various shapes and positions in the diffraction patterns from the icosahedral phase, violating the parity rule for simple icosahedral (SI) symmetry. In the process of annealing treatment, the diffuse spots also evolve in the centre of the arcs and become sharp. These extra diffuse spots change the symmetry of the quasilattice from $P$-type to $F$-type. The ordered and disordered structures in quasicrystal have been linked to the ordered and disordered structures present in the crystalline $\alpha(\mathrm{Al}-\mathrm{Mn}-\mathrm{Si})$ and $\alpha(\mathrm{Al}-\mathrm{Fe}-\mathrm{Si})$ alloys.
\end{abstract}

Keywords. Quasicrystals; rational approximants; ordering.

\section{Introduction}

While studying the metastable phase formation in rapidly solidified aluminiumtransition metal alloys, Shechtman et al (1984) discovered the icosahedral phase which is also known as an icosahedral quasicrystal (QC) (Levine and Steinhardt 1984). This phase shows 5-fold, 3-fold and 2-fold rotational symmetries corresponding to the icosahedral point group $(m \overrightarrow{53})$ in reciprocal space. The corresponding Bravais lattices compatible with this point group have been identified in 6-dimensional (6D) space. Rokhsar et al (1987) have shown that there are three possible cubic space lattices in 6D space similar to those in $3 \mathrm{D}$ space. These are (i) simple cubic ( $\mathrm{SC}$ ), (ii) body centred cubic (BCC) and (iii) face centred cubic (FCC). The 6D cubic lattices i.e. SC, FCC, BCC after projection on irrational orientation onto $3 \mathrm{D}$ space can give rise to simple icosahedral (SI), face centred icosahedral (FCI) and body centred icosahedral (BCI) structures respectively.

Most quasicrystals belong to the SI model. However, quasicrystals in $\mathrm{Al}-\mathrm{Fe}-\mathrm{Cu}$ (Ishimasa et al 1988), Al-Cu-Cr, Al-Cu-Mn, Al-Cu-Fe (Ebalard and Spaepen 1989, 1990) and Al-Pd-Mn (Tsai et al 1990) alloys conform to the FCI model in real space and $\mathrm{BCI}$ model in reciprocal space. $\mathrm{FCI}$ ordering has been reported for $\mathrm{Mg}$-base alloys by Nikura et al (1993). Ishimasa (1995) recently reported that the FCI quasicrystal in $\mathrm{Al}-\mathrm{Mn}-\mathrm{Pd}$ alloy orders further at low temperatures to give rise to a SI quasicrystal. This is also described by him using a six dimensional diamond lattice. The lattice parameters of FCI, SI and diamond lattices are related to each other.

Many other subtle features in the diffraction patterns of the icosahedral phase have been recorded in the literature. The issues raised by these anomalies are critical, as they can resolve the controversy surrounding the concept of quasicrystals (Mukhopadhyay 1990). Mukhopadhyay et al (1987) observed the occurrence of diffuse intensity in electron diffraction in $\mathrm{Mg}-\mathrm{Al}-\mathrm{Zn}$ and $\mathrm{Al}-\mathrm{Mn}$ alloys. Denoyer et al (1987) showed that 
diffuse intensity exists in X-ray diffraction patterns from $\mathrm{Al}-\mathrm{Li}-\mathrm{Cu}$ alloys. The appearance of the diffuse intensity was reported by Mandal et al (1991) in Fe-Ti (also to be seen in the diffraction pattern of Dong et al 1986). Kelton et al (1988) and Bhaskaran et al $(1993,1994)$ in Ti-Mn and Swamy et al (1989) in Al-Cr and also Mukhopadhyay et al (1992) in Al-Fe-W. By systematic heat treatment Mukhopadhyay et al (1989) have shown that the arcs in $\mathrm{Al}-\mathrm{Mn}$ alloys lead to an ordering transformation. This was also observed by Ebalard and Spaepen (1990) and Koster and Liu (1993). Hiraga et al (1989) found the order-disorder transition temperature in $\mathrm{Al}-\mathrm{Cu}-\mathrm{Ru}$ system. It is of interest to mention that Tsai et al (1990) noticed the ordering transition by replacing Al by $\mathrm{Pd}$ in $\mathrm{Al}-\mathrm{Pd}-\mathrm{Mn}$ alloys. The aim of the present investigation is to probe the evolution of ordering in quasicrystals and to establish a link with the ordering in related crystalline prototype structures of $\alpha(\mathrm{Al}-\mathrm{Mn}-\mathrm{Si})$ and $\alpha(\mathrm{Al}-\mathrm{Fe}-\mathrm{Si})$.

\section{Diffuse intensity and superlattice spots in diffraction patterns}

The 2-fold diffraction pattern (figure 1a) from as quenched quasicrystalline phase in Al $-10 \% \mathrm{Mn}$ reflects the disordered SI symmetry. However, on aging diffuse intensity appears in the diffraction patterns (figure $1 \mathrm{~b}$ ) which shows arcs of diffuse intensity in the 2-fold patterns from $\mathrm{Al}-10 \% \mathrm{Mn}$, aged at $325^{\circ} \mathrm{C}$ for $45 \mathrm{~min}$. Figure $1 \mathrm{c}$ shows 2 -fold pattern exhibiting the extra diffuse spots or weak intensity spots along 3-fold and 5-fold directions. On the other hand, 5-fold and 3-fold zone axis patterns do not show any observable change and the diffuse intensities are absent in these patterns. A detailed analysis of the location of the arcs of diffuse intensities indicates that they occur only along odd parity directions. If we consider the centres of these intensity distribution, $a$ ' $\tau$ ' inflation scheme describes both the fundamental spots and the centres of these diffuse arcs (figure 1b). Further analysis indicates the presence of diffuse spots in the centres of the arcs (marked by arrow). The co-existence of the diffuse arcs and diffuse spots is noticed for the first time after careful analysis of the diffraction patterns. In figure 1c the diffuse arcs disappear, while the diffuse spots in the centre become intense and sharp enough to be identified. These extra spots violate the parity rule for the SI symmetry in the diffraction pattern. The extra spots along 5-fold direction can be indexed based on $\mathrm{BCI}$ symmetry and this confirms the parity rule for $\mathrm{FCI}$ in real space. The diffraction pattern from the ordered quasicrystal $(\mathrm{Al}-\mathrm{Fe}-\mathrm{Cu}$ ) has been displayed in figure $1 \mathrm{~d}$ to compare with figure $\mathrm{IC}$. From the analysis of these diffraction patterns, it appears that the degree of long range FCI ordering is zero in figure 1a and one in figure $1 \mathrm{~d}$, and it is intermediate in figures $1 \mathrm{~b}$ and $\mathrm{c}$. In other words, figures $1 \mathrm{~b}$ and $\mathrm{c}$ can be considered as partially ordered states while the degree of ordering is more in figure $1 \mathrm{c}$ as there are no diffuse arcs present. However, in $\mathrm{Al}-10 \% \mathrm{Mn}$ system, to attain fully ordered state or perfect long range ordering is inherently difficult. The intensity of the superlattice spots is not as bright as those in $\mathrm{Al}-\mathrm{Fe}-\mathrm{Cu}$. Experimental results indicate that the replacement of $\mathrm{Al}$ by transition metal (other than $\mathrm{Mn}$; for example $\mathrm{Pd}$ ) plays an important role in the ordering process. In $\mathrm{Al}-10 \% \mathrm{Mn}$ as the transition element is less, the perfect long range ordering may not be possible.

Figures $2 \mathrm{a}$ and $\mathrm{b}$ show two-fold diffractions from disordered and ordered $\alpha(\mathrm{Al}-\mathrm{Mn}-$ $\mathrm{Si}$ ) crystals. The former is isostructural to $\alpha$ ( $\mathrm{Al}-\mathrm{Fe}-\mathrm{Si})$ phase, being $\mathrm{BCC}$. By comparing both the diffraction patterns, one can find out the extra spots in figure $2 \mathrm{~b}$. Though the point group symmetry is the same for both the cases, the Bravais lattices are 


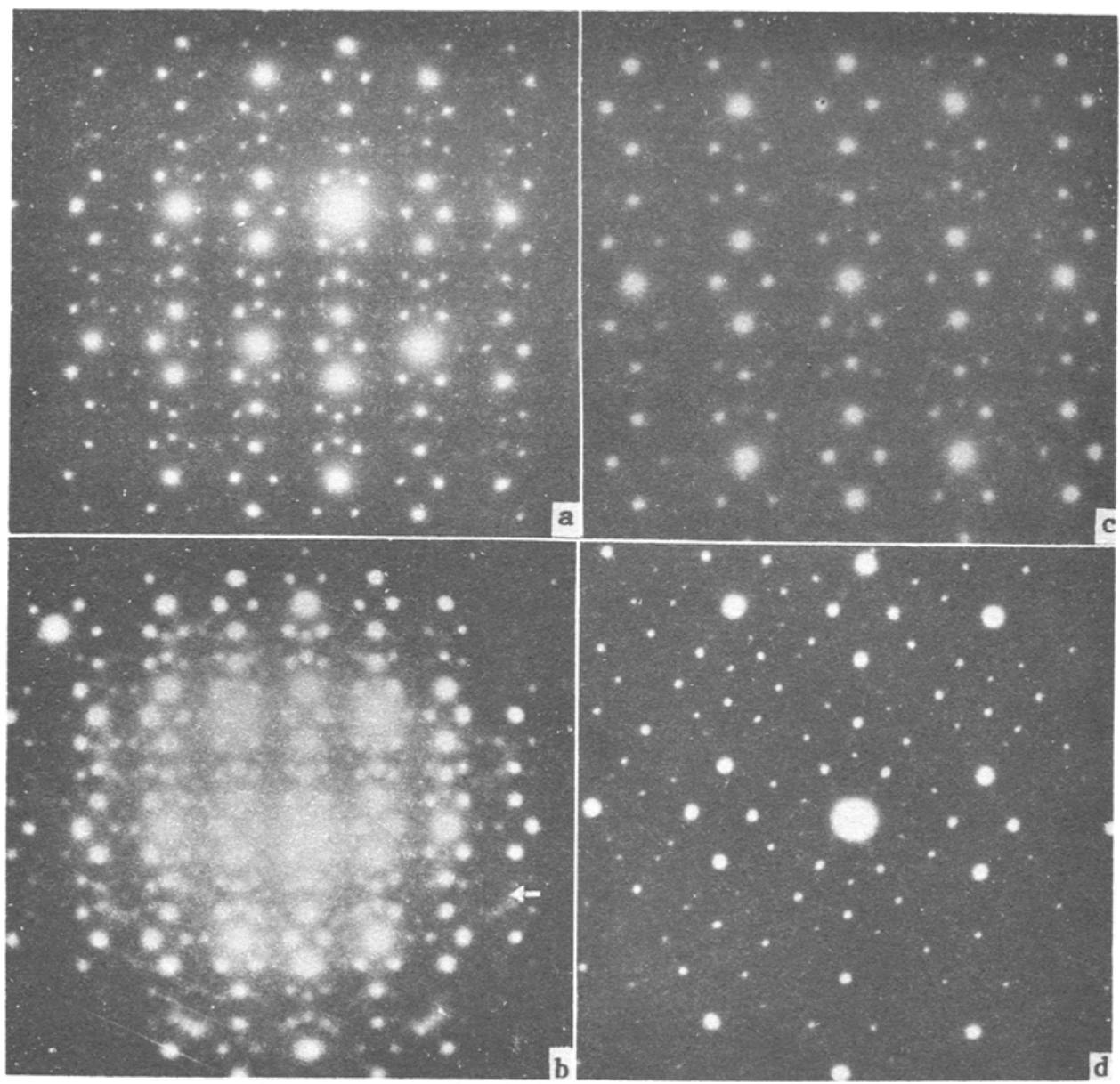

Figure 1. Two fold diffraction patterns from (a) disordered Al-10\% Mn quasicrystals, (b) partially ordered showing the diffuse arcs and also diffuse spots obtained from $\mathrm{Al}-10 \% \mathrm{Mn}$ QC, aged at $325^{\circ} \mathrm{C}$ for $45 \mathrm{~min}$, (c) partially ordered $\mathrm{Al}-10 \% \mathrm{Mn}$ alloy, aged at $325^{\circ} \mathrm{C}$ for $45 \mathrm{~min}$ (but from a different region of the sample), showing the diffuse spots and (d) fully ordered QC from $\mathrm{Al}-\mathrm{Fe}-\mathrm{Cu}$ system.

different. The diffraction pattern from ordered $\alpha(\mathrm{Al}-\mathrm{Mn}-\mathrm{Si})$ confirms $\mathrm{SC}$ symmetry whereas the other one has $\mathrm{BCC}$ symmetry. However, both are related by the ordering reaction similar to $\mathrm{CsCl}$-type structure. This can be compared with the computed patterns obtained from $1 / 1$ projection of $6 \mathrm{D} \mathrm{SC}$ and $6 \mathrm{D} \mathrm{FCC} \mathrm{structures.} \mathrm{The} \mathrm{similarity}$ between figures $2 a$ and $b$ and $3 a$ and $b$ can be noted. This will be discussed further in the later section.

\section{Face centred ordering $(P \rightarrow F)$}

At present from the systematic experimental data it can be understood that the arcs of diffuse intensity and the subsequent appearance of spots with $\tau$ inflation are related in 


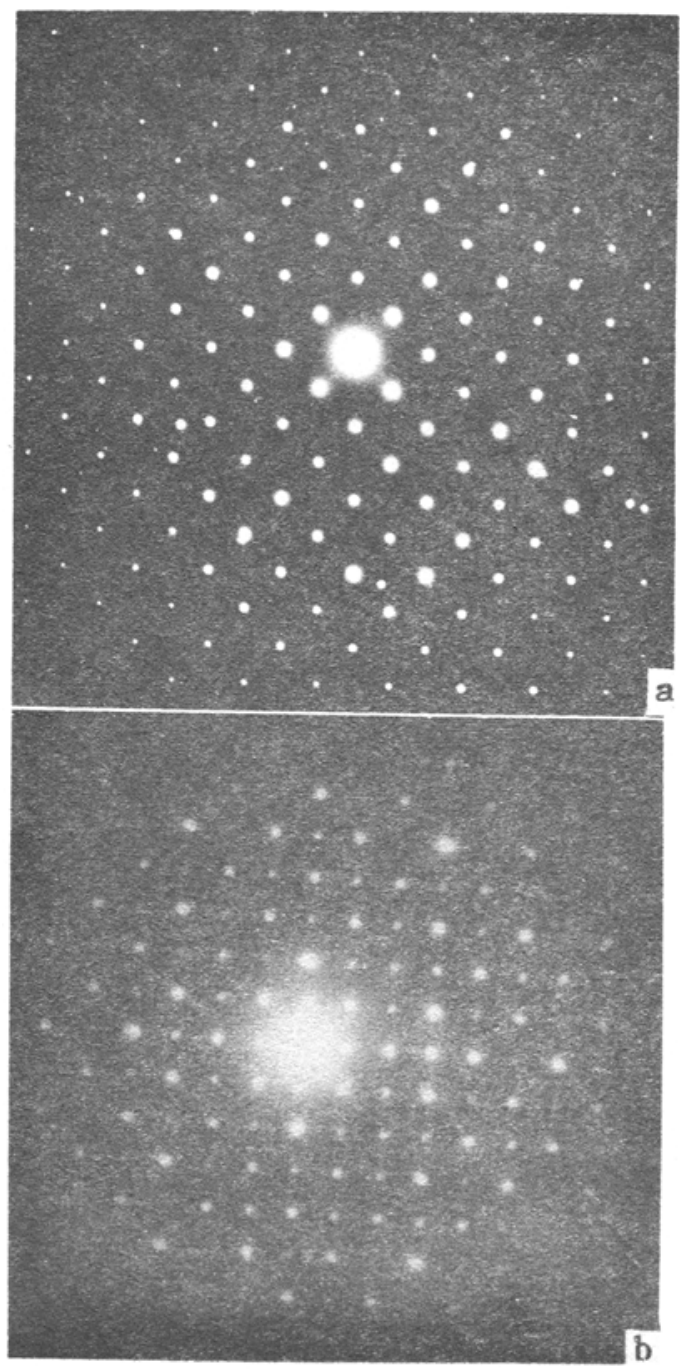

Figure 2. The 2-fold crystalline patterns from (a) disordered $\mathrm{BCC}$ approximant: $\mathrm{Al}-\mathrm{Mn}-\mathrm{Si}$ and (b) ordered SC approximant: $\alpha$ (AlMnSi) structures.

the transition metal systems for which $\mathrm{Al}-\mathrm{Mn}-\mathrm{Si}$ is a prototype. A possible model has been put forward by Cahn (1987) and Henley (1988) independently. According to this model the superlattice ordering will take place in SI quasilattice and it will lead to FCI in real space. Therefore, the disorder-order reaction can be realized in reciprocal space through diffuse spots in the centre of the diffuse arcs:

$$
\text { SI }\left(a_{\mathrm{R}}\right) \rightarrow \text { diffuse spots } \rightarrow \text { BCI }\left(2 a_{\mathrm{R}}\right) .
$$

The present ordered structure in $6 \mathrm{D}$ can be imagined as $\mathrm{NaCl}$ type structure. On disordering, the cell parameter is reduced to half the original size. The odd parity spots represent the superlattice positions and the even parity describes the fundamental positions. The quasilattice constant of the ordered $\mathrm{QC}$ is twice the previous one. 

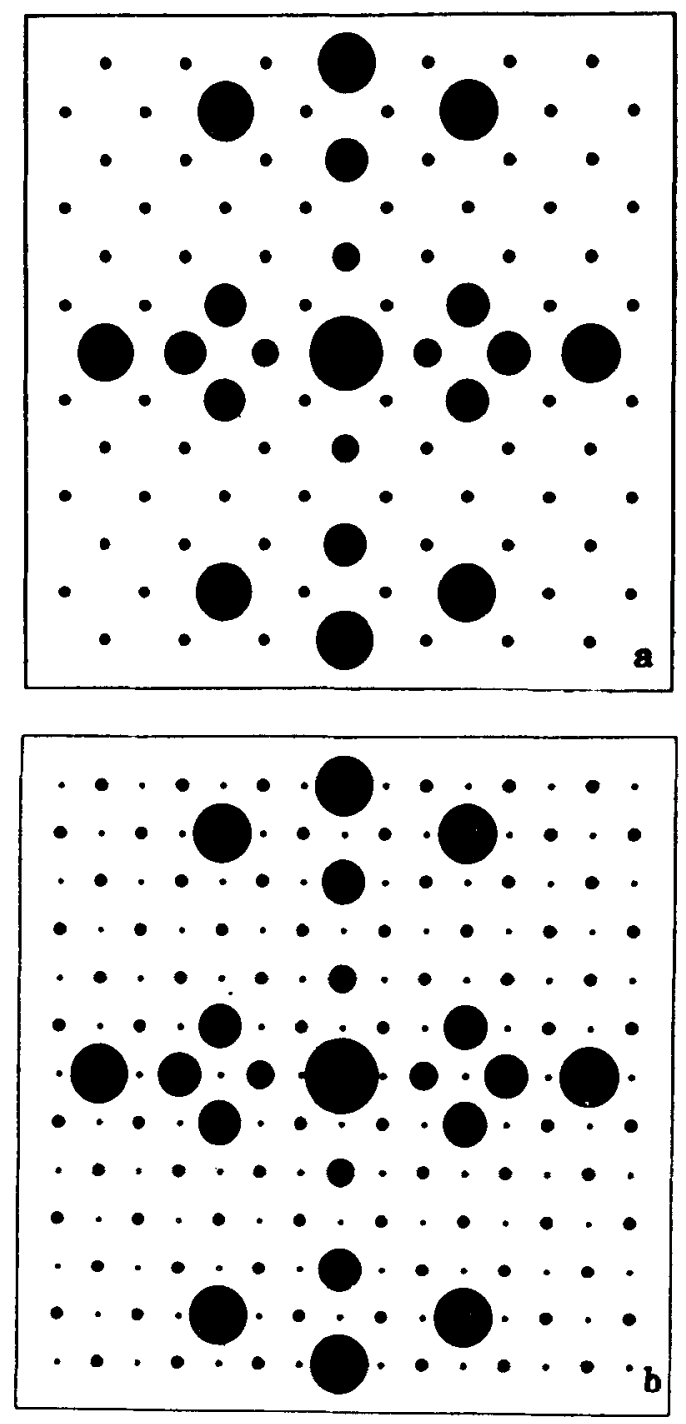

Figure 3. Computed diffraction patterns for (a) disordered $1 / 1$ structure and (b) ordered $1 / 1$ structures. These have been generated from $6 \mathrm{D}$ reciprocal spaces. The intensity reflects the intrinsic structure factor. Geometrical structure factor has not been considered.

It is of interest to note that in some of the $\mathrm{Ti}$ - and $\mathrm{Mg}$-based quasicrystals where the diffuse arcs are similar to those of Al-Mn system, the superlattice spots could not be observed. It has been shown by Kelton and Gibbons (1992) that diffuse arcs do not vanish and are present even in the rational approximant structure of the Ti-base quasicrystals. This rational approximant has the same crystal structure as $\alpha$-AlMnSi. A disordered rational approximant has not been reported in Ti-base alloys.

In the work on $\mathrm{Al}-\mathrm{Li}-\mathrm{Mg}$ alloys by Nikura et al (1993) an ordered phase was observed, though no arcs or diffuse intensity was reported. As the rational approximant for this alloy would be a Frank-Kasper phase, the explanation that the ordering of the 
quasicrystal is related to the ordering in the rational approximant will not apply to this alloy. Further studies of this alloy are warranted.

\section{Link between the ordered and disordered crystals and quasicrystals}

We propose that the face centred ordering which has been discussed in earlier section is intimately linked to the ordered and disordered structure in crystalline $\alpha$ (AIMnSi) and $\alpha(\mathrm{AlFeSi})$ alloys. The $\alpha(\mathrm{AlMnSi})$ is described by a SC structure with space group $P m \overline{3}$ and $\alpha(\mathrm{Al}-\mathrm{Fe}-\mathrm{Si})$ is BCC with space group $\operatorname{Im} \overline{3}$ (Cooper and Robinson 1966; Cooper 1967). In fact, $\alpha$ (AlMnSi) can be treated as an ordered form of $\alpha$ (AlFeSi). While $\mathrm{Mn}$ and $\mathrm{Al}$ sites in the former are related, some sites in the latter case are unpaired making the structure disordered (Mandal 1990). The order-disorder reaction linking these two structures has been observed by Legresy et al (1986) in $\mathrm{Al}-\mathrm{Fe}-\mathrm{Si}$ alloy by electron beam heating.

It is well known that rational approximant structure can be generated by projection from hyper dimensional structure onto rational orientation. Thus, $\alpha(\mathrm{Al}-\mathrm{Mn}-\mathrm{Si})$ is a $1 / 1 \mathrm{SC}$ approximant and $\alpha(\mathrm{Al}-\mathrm{Fe}-\mathrm{Si})$ is $1 / 1 \mathrm{BCC}$ approximant. $1 / 1 \mathrm{SC}$ approximant is generated from ordered FCI quasicrystal and $1 / 1 \mathrm{BCC}$ approximant can be generated from disordered SI quasicrystals. Figure $3(a, b)$ shows the $[100]$ zone axis corresponding to BCC and SC structures. The extra superlattice spots can be visualized from the diffraction patterns. They also correspond to the patterns from $\alpha$ ( $\mathrm{Al}-\mathrm{Fe}-\mathrm{Si})$ and $\alpha(\mathrm{Al}-\mathrm{Mn}-\mathrm{Si})$ respectively. It is thus possible to infer that the disordered and ordered structure seen in 3D has its analog in $6 \mathrm{D}$ as per the scheme shown in figure 4. This illustrates the link between the order-disorder transformation in quasicrystals and crystals. It is thus possible to observe the ordering reaction by suitable annealing or alloying treatment. A recent paper by Donnadieu et al (1994) lends further authority to the above suggestion. They studied a set of Al-Mn-Fe-Si alloys, where the $\mathrm{Mn} / \mathrm{Fe}$ ratio was varied. In the crystals for low values of $\mathrm{Mn} / \mathrm{Fe}$ selected area diffraction patterns showed a noticeable diffuse scattering due to short range ordering. As the $\mathrm{Mn} / \mathrm{Fe}$ ratio is increased the diffuse rings are replaced by weak superlattice spots.

It is pertinent to point out that our identification of the $1 / 1$ rational approximant for the FCI quasicrystal is at variance with that of Khare et al (1995). In an investigation on $\mathrm{Al}-\mathrm{Cu}-\mathrm{Cr}$ alloys they found crystalline bcc $(a=12.6 \AA$, disordered $)$, simple cubic ( $a=12 \cdot 60 \AA$, ordered) and another bcc $(a=25 \cdot 20 \AA)$ phases. They identified the first two phases as $1 / 1$ rational approximants of SI quasicrystal and the second bcc phase

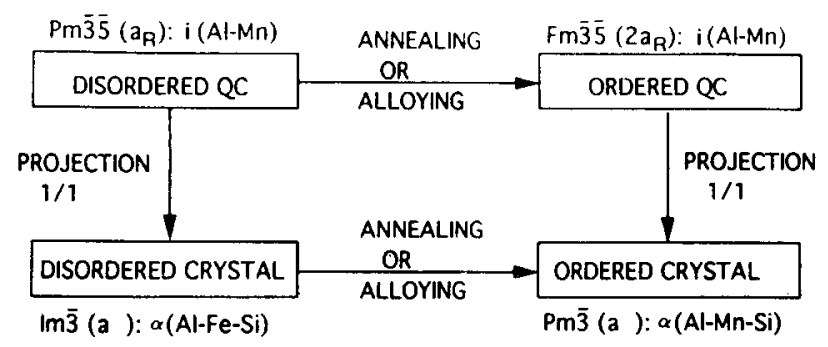

Figure 4. Schematic diagram showing the link between disordered and ordered forms of quasicrystals and crystals. 
with twice the lattice parameter as the $1 / 1$ rational approximant of the FCI quasicrystal. It is perhaps likely that this rational approximant structure corresponds to the second ordering reaction observed by Ishimasa (1995). Further study is required to confirm this speculation.

\section{Acknowledgements}

The authors would like to thank Dr Alok Singh, Dr O Prakash and Dr M V Ravichandran for many useful discussions. Mr A K Srivastava is thanked for providing a diffraction pattern. The work has derived support from Indo-US scientific cooperation program and DST project on quasicrystals.

\section{References}

Bhaskaran T A, Krishnan R V, Ranganathan S and Kelton K F 1993 Metastable microstructure (eds) D Banerjee and L A Jacobson (New Delhi: Oxford and IBH Publishing Co Pvt Ltd) p. 93

Bhaskaran T A, Krishnan R V and Ranganathan S 1994 Bull. Mater. Sci. 17795

Cahn J W 1987 private communication, cited in Chattopadhyay K and Mukhopadhyay N K 1987 Mater. Sci. Forum 22 24639

Cooper M 1967 Acta Crystallogr. 231106

Cooper M and Robinson K 1966 Acta Crystallogr. 20614

Denoyer F. Heter G. Lambert M, Lang J M and Sainfort P 1987 J. Phys. (Paris) 481357

Donnadieu P, Lapasset G and Sanders T H 1994 Philos. Mag. 70319

Dong C, Hei Z K, Wang L B. Song Q H, Wu Y K and Kuo K H 1986 Scr. Metall. 201155

Ebalard S and Spaepen F S 1989 J. Mater. Res. 539

Ebalard S and Spaepen F S 1990 J. Mater. Res. 562

Henley C L 1988 Philos. Mag. Lett. $\mathbf{5 8} 87$

Hiraga K. Hirabayashi M, Tsai A P, Inoue A and Masumoto T 1989 Philos. Mag. Lett. 60201

Ishimasa 11995 Philos. Mag. Lett. 7165

Ishimasa I, Fukano Y and Tsuchimori M 1988 Philos. Mag. Lett. 58157

Kelton K F, Gibbon P C and Sabes P N 1988 Phys. Rev. B38 7810

Kelton K F and Gibbons P C 1992 Philos. Mag. B66 639

Khare V. Lalla N P. Tiwari R S and Srivastava O N 1995 J. Mater. Res. 81905

Koster U and Liu W 1993 Phase Transitions 144137

Legresy J M. Audier M, Simon J P and Guyot P 1986 Acta Metall. 341759

Levine D and Steinhardt P J 1984 Phys. Rev. Lett. 532477

Mandal P. Tiwari R S and Srivastava O N 1991 Philos. Mag. Lett. 63617

Mukhopadhyay N K 1990 Some aspects of synthesis, structure and stability of quasicrystals. PhD Thesis. IISc. Bangalore

Mukhopadhyay N K, Ranganathan S and Chattopadhyay K 1987 Philos. Mag. Lett. 56121

Mukhopadhyay N K, Ranganathan S and Chattopadhyay K 1989 Philos. Mag. Lett. 60207

Mukhopadhyay N K, Weatherly G C, Lloyd D J and Embury J D 1992 Scr. Metall. et Mater. 27111

Nikura A, Tsai A P, Inoue A, Masumoto T and Yamamoto A 1993 Jpn J. Appl. Phys. 32 L1160

Rokhsar D S, Mermin N D and Wright D 1987 Phys. Rev. B35 5487

Shechtman D, Blech I, Gratias D and Cahn J W 1984 Phys. Rev. Lett. 531951

Swamy V T. Ranganathan S and Chattopadhyay K 1989 J. Mater. Res. 4539

Tsai A P. Inoue A and Masumoto T 1988 Jpn J. Appl. Phys. 27 L1505

Tsai A P. Inoue A. Yokoyama Y and Masumoto T 1990 Philos. May. Lett. 619 